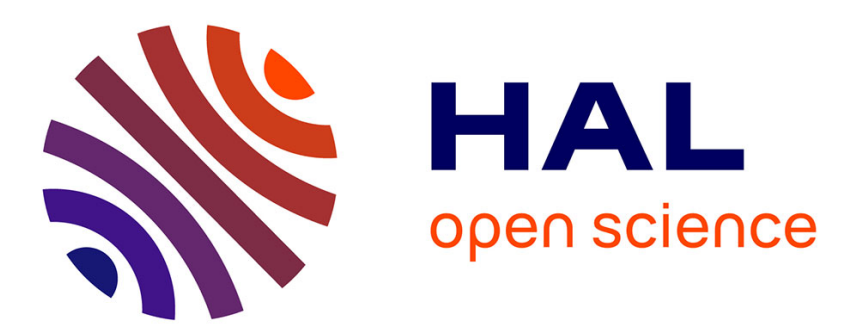

\title{
Nonperiodicity of the flow within the gap of a thermoacoustic couple at high amplitudes
}

\author{
Arganthaël Berson, Philippe Blanc-Benon
}

\section{To cite this version:}

Arganthaël Berson, Philippe Blanc-Benon. Nonperiodicity of the flow within the gap of a thermoacoustic couple at high amplitudes. Journal of the Acoustical Society of America, 2007, 122 (4), pp.EL122-EL127. 10.1121/1.2771370 . hal-00707894

\section{HAL Id: hal-00707894 \\ https://hal.science/hal-00707894}

Submitted on 13 Jun 2012

HAL is a multi-disciplinary open access archive for the deposit and dissemination of scientific research documents, whether they are published or not. The documents may come from teaching and research institutions in France or abroad, or from public or private research centers.
L'archive ouverte pluridisciplinaire HAL, est destinée au dépôt et à la diffusion de documents scientifiques de niveau recherche, publiés ou non, émanant des établissements d'enseignement et de recherche français ou étrangers, des laboratoires publics ou privés. 


\title{
Nonperiodicity of the flow within the gap of a thermoacoustic couple at high amplitudes
}

\author{
Arganthaël Berson and Philippe Blanc-Benon \\ LMFA, UMR CNRS 5509, Ecole Centrale de Lyon, 69134 Ecully Cedex, France \\ arganthael.berson@ec-lyon.fr,philippe.blanc-benon@ec-lyon.fr
}

\begin{abstract}
The flow inside a thermoacoustic couple is investigated experimentally using particle image velocimetry. Measurements show the oscillation of the shear layers flowing out of a single stack, thus forming an asymmetric vortex street at high driving amplitudes. Development of vortices is also observed within the gap of a thermoacoustic couple. It causes the flow not to repeat from one acoustic period to another. The nonperiodicity of the flow will lead to unsteady heat transfer between the stack and heat exchangers and to the oscillation of the cooling load.

(C) 2007 Acoustical Society of America

PACS numbers: 43.35.Ud [TM]

Date Received: June 23, 2007

Date Accepted: July 23, 2007
\end{abstract}

\section{Introduction}

The design of appropriate heat exchangers is a crucial issue for the development of efficient thermoacoustic systems. Recent numerical studies (Marx, 2003; Besnoin and Knio, 2004) have shown that heat transfer through the gap between a thermoacoustic stack and a heat exchanger is strongly coupled with the flow patterns in this area. The improvement of thermoacoustic system performances requires a better understanding of such flows, especially at high amplitudes when nonlinear effects become more important (Poese and Garrett, 2000). The flow behind a single stack has been investigated experimentally by Wetzel and Herman (2000), Duffourd (2001), and Berson et al. (2007), showing vortex generation close to the end of the stack plates at low amplitude. Such flow is responsible for minor losses and energy dissipation as was explored by Waxler (2001), Wakeland and Keolian (2002), and Smith and Swift (2003).

After a brief description of the experimental setup, this study presents particle image velocimetry (PIV) measurements behind a single stack. Vortex shedding at high acoustic pressure level is reported and dimensionless numbers are estimated. The last part investigates the flow between two adjacent stacks separated by a gap as a model for a stack coupled to a heat exchanger. The results show that, at high amplitudes, vortex shedding causes the flow in the gap not to be repeatable from one acoustic period to another. A direct consequence will be the oscillation of the cooling load, as was demonstrated in the numerical studies by Besnoin (2001).

\section{Experimental setup}

\subsection{The thermoacoustic refrigerator}

Experiments are conducted in a quarter-wavelength standing-wave thermoacoustic refrigerator. The resonator is a closed cylindrical tube of length $L=150 \mathrm{~mm}$ and diameter $2 R=30 \mathrm{~mm}$. A 25-mm-long part of the resonator is made of specially polished glass in order to ensure high quality optical access for PIV measurements. The system is driven by a GELEC EDM8760F loudspeaker that is connected to the resonator by an exponential adaptation horn. The fluid inside the resonator is air at atmospheric pressure. The driver delivers acoustic pressure levels up to $P_{\mathrm{ac}}=5000 \mathrm{~Pa}$ at the acoustic resonance frequency $f_{\text {res }} \approx 460 \mathrm{~Hz}$. Acoustic pressure is monitored by a 1/4 in. Bruel \& Kjaer microphone flush mounted at the closed end of the resonator.

Thermoacoustic stacks are hand-made of thin glass plates separated by plastic spacers. Three different types of stacks have been built, which are hereafter referred to as stacks A, B, 
Table 1. Characteristics of the different stack configurations. $l$ is the plate length, $y_{0}$ is the plate spacing, $e_{0}$ is the plate thickness, $\mathrm{BR}=y_{0} /\left(y_{0}+e_{0}+S_{\text {solid }}\right)$ is the blockage ratio, $S_{\text {solid }}$ is the area of the support and $\delta_{\nu}=\sqrt{\nu /\left(\pi f_{\text {res }}\right)}$ is the viscous boundary layer thickness, with $\nu$ the kinematic viscosity of the fluid.

\begin{tabular}{lccccc}
\hline \hline Stack & A & B & C & D & E \\
\hline$l(\mathrm{~mm})$ & 18 & 18 & 24 & 25 & $\ldots$ \\
$y_{0}(\mathrm{~mm})$ & 0.41 & 0.27 & 0.42 & 1 & 0.93 \\
$e_{0}(\mathrm{~mm})$ & 0.17 & 0.17 & 0.17 & 1 & 0.47 \\
$\mathrm{BR}$ & 0.39 & 0.34 & 0.62 & 0.5 & 0.66 \\
$f_{\text {res }}(\mathrm{Hz})$ & 461 & 455 & 464 & 210 & 110 \\
$\delta_{\nu}(\mathrm{mm})$ & 0.10 & 0.10 & 0.10 & 0.15 & 0.21 \\
\hline \hline
\end{tabular}

and C. Their geometrical characteristics are summarized in Table 1. Data for stacks D and E, which are also reported in Table 1, are taken from the experimental work presented in Berson et al. (2007) and from the numerical simulations of Besnoin and Knio (2004). Further details about configurations $\mathrm{D}$ and $\mathrm{E}$ can be found in these references. In the present configurations, the hot end of the stack is located at $x_{h}=65 \mathrm{~mm}\left(x_{h}\right.$ being the distance from the closed end of the resonator). Additional tests were performed with stack A located at $x_{h}=115 \mathrm{~mm}$. The investigation of the flow between two coupled stacks refers to two type-A stacks. In this case, a first stack is located at $x_{h}=65 \mathrm{~mm}$ and a second one is placed just behind and closer to the closed end of the resonator. Care was taken to make the plates of both stacks as parallel as possible with respect to each other. Adequate plastic spacers separate both stacks, ensuring gap width of approximately $g=0.7,1.3$, or $2.3 \mathrm{~mm}$ between the plate ends. Although these locations are not optimal for the thermoacoustic effect, we believe that the general behavior of the flow hardly depends on the temperature gradient along the stack and is mostly aerodynamical.

\subsection{The PIV measuring system}

Measurements are performed with a LaVision ${ }^{\mathrm{TM}}$ digital camera and a $12 \times$ Navitar zoom lens. The measurement area is in a plane perpendicular to the stack plates and parallel to the resonator axis. Its size ranges from $10 \times 8 \mathrm{~mm}^{2}$ down to $2.5 \times 3 \mathrm{~mm}^{2}$. A dual-resonator Nd:YAG laser illuminates the flow field that is seeded with droplets of parrafin oil smoke. Data acquisition is synchronized with the signal driving the loudspeaker and we perform phase averaging to overcome the sampling frequency limitations of the acquisition system. An acoustic period is decomposed in 32 equally spaced phases $\Phi$, where $\Phi=0^{\circ}$ corresponds to the instant when acoustic pressure is zero and velocity is maximal, the flow being directed toward the loudspeaker. For each selected phase, velocity fields are averaged over 50 instant measurements. A more detailed description and the validation of this PIV measurement technique can be found in Duffourd (2001), Blanc-Benon et al. (2003), and Berson et al. (2007). For analysis purposes, we calculate two-dimensional vorticity fields $\Omega$ and $\Gamma_{2}=2 / \pi$ contours from phase-averaged velocity measurements. $\Gamma_{2}$ function is a nondimensional quantity defined at a point $M$ of the space as

$$
\Gamma_{2}(\mathbf{x})=\frac{1}{S} \iint_{M^{\prime} \in S} \sin \theta \mathrm{d} x_{i}^{\prime} \mathrm{d} x_{j}^{\prime},
$$

with $S$ the integration domain, centered on $M . M^{\prime}$ belongs to $S$ and its coordinates are $\mathbf{x}^{\prime} . \theta$ is the angle between $\mathbf{x}^{\prime}-\mathbf{x}$ and $\mathbf{u}\left(M^{\prime}\right)-\mathbf{u}(M), \mathbf{u}(M)$ being the velocity at coordinate $M$. Subscripts $i$ and $j$ refer to the vector components in the plane. $\Gamma_{2}$ function is comprised between $2 / \pi$ and 1 when the flow is locally dominated by rotation, thus allowing one to detect vortex boundaries. A complete description of $\Gamma_{2}$ function is given in Graftieaux et al. (2001). 


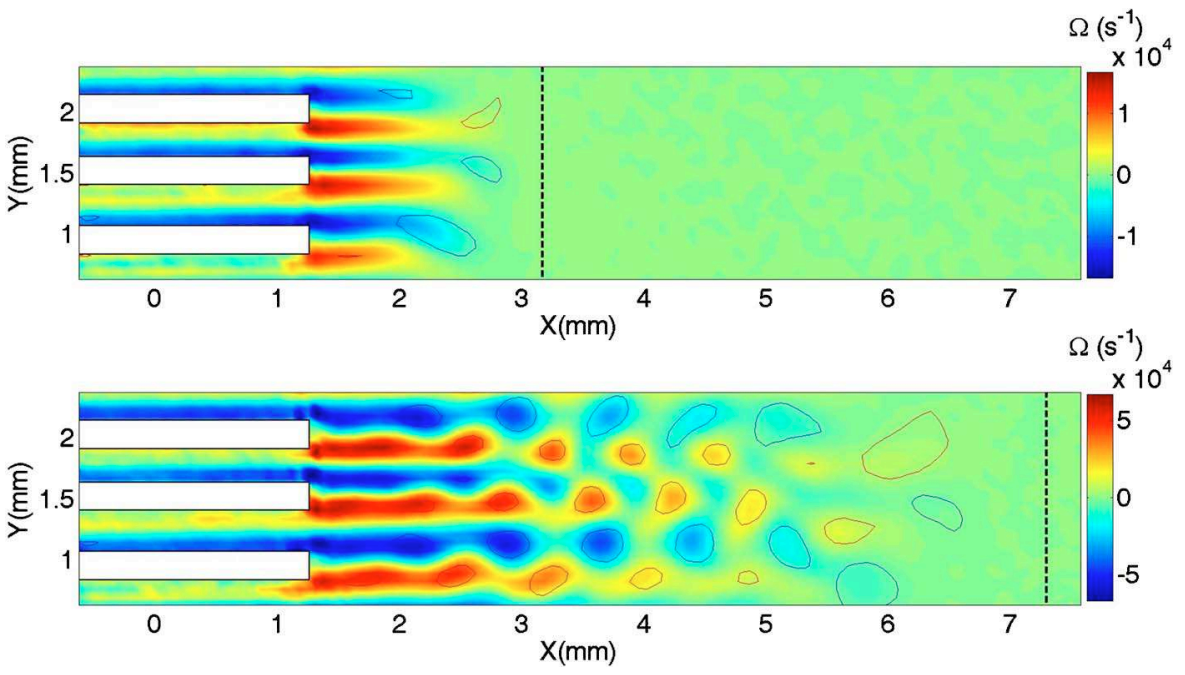

Fig. 1. (Color online) Vorticity fields $\Omega\left(\mathrm{s}^{-1}\right)$ and $\Gamma_{2}=2 / \pi$ contours behind stack A. $\Phi=225^{\circ}$. Top panel: $P_{\text {ac }}$ $=1000 \mathrm{~Pa}$, bottom panel: $P_{\mathrm{ac}}=4000 \mathrm{~Pa}$. The black dashed line represents the distance $2 d_{\mathrm{ac}}$ away from the plate edge. Note that the color scales are different for improved readability.

\section{Results and discussion}

\subsection{Oscillation of the flow behind a single stack at high amplitudes}

The flow field behind a single stack has been previously investigated experimentally by Duffourd (2001) and Berson et al. (2007) for low acoustic pressure level configurations $\left(P_{\mathrm{ac}}\right.$ $\leqslant 2000 \mathrm{~Pa}$ ). Vortex shedding was observed in Berson et al. (2007) in the case of thick stack plates $\left(e_{0} / \delta_{\nu} \approx 7\right)$. With more practical and thinner plates $\left(e_{0} / \delta_{\nu}<2\right)$, as is the case here, no vortex shedding was observed in the range of acoustic velocity investigated at the time.

Figure 1 shows vorticity fields and $\Gamma_{2}=2 / \pi$ contours of the flow behind stack A at phase $\Phi=225^{\circ}$ for $P_{\mathrm{ac}}=1000 \mathrm{~Pa}$ and $P_{\mathrm{ac}}=4000 \mathrm{~Pa}$. Measurements are phase-averaged over 50 images. A movie of the complete acoustic period is available in $\mathrm{Mm}$. 1. Note that due to a slight shift in the laser alignment with the stack plates, the flow within the channels of the stack is not measured accurately. Such flow is detailed in Duffourd (2001) and Berson et al. (2007). The measurements of the flow behind the stack are not affected by this shift.

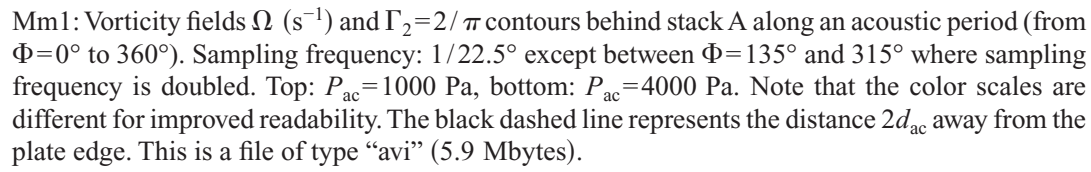

Acoustic velocity amplitudes $u_{\mathrm{ac}}\left(x_{h}\right)$ corresponding to $P_{\mathrm{ac}}=1000 \mathrm{~Pa}$ and $P_{\mathrm{ac}}$ $=4000 \mathrm{~Pa}$ at the stack location $x_{h}=115 \mathrm{~mm}$ are $u_{\mathrm{ac}}\left(x_{h}\right)=2 \mathrm{~m} \mathrm{~s}^{-1}$ and $u_{\mathrm{ac}}\left(x_{h}\right)=8 \mathrm{~m} \mathrm{~s}^{-1}$, respectively. At $P_{\mathrm{ac}}=1000 \mathrm{~Pa}$, vorticity layers extend out of the stack during the expulsion phases (when the fluid is flowing out of the hot side of the stack), thus prolongating the oscillating boundary layers that develop along the stack plates. At $P_{\mathrm{ac}}=4000 \mathrm{~Pa}$, we observe that the vorticity layers outside the stack oscillate and generate asymmetric streets of counter-rotating vortex pairs shedding away from the stack. In the range of acoustic velocities studied here, oscillation of the vorticity layers occurs only during the phases when the fluid flows out of the stack and decelerates due to the acoustic standing wave (from $\Phi=180^{\circ}$ to $\Phi=270^{\circ}$ ). Note that gravity is directed perpendicular to the measurement plane so that buoyancy should not be responsible for these oscillations. We also notice that, in all the configurations tested in this study, the vor- 
Table 2. Reynolds number and formation number at onset of vortex shedding and Strouhal numbers based on various characteristic lengths for different stack configurations.

\begin{tabular}{lcccccc}
\hline \hline Stack & $\begin{array}{c}\mathrm{A} \\
\left(x_{h}=65 \mathrm{~mm}\right)\end{array}$ & $\begin{array}{c}\mathrm{A} \\
\left(x_{h}=115 \mathrm{~mm}\right)\end{array}$ & $\mathrm{B}$ & $\mathrm{C}$ & $\mathrm{D}$ & $\mathrm{E}$ \\
\hline $\mathrm{Re}_{\text {onset }}$ & 94 & 99 & 108 & 60 & 102 & $\ldots$ \\
$T_{\text {onset }}^{*}$ & 6.4 & 6.8 & 9.4 & 6.0 & 11.2 & $\ldots$ \\
$\mathrm{St}_{\delta_{\nu}}$ & 0.12 & 0.12 & 0.14 & 0.11 & 0.07 & 0.10 \\
$\mathrm{St}_{e_{0}}$ & 0.20 & 0.20 & 0.25 & 0.19 & 0.44 & 0.23 \\
$\mathrm{St}_{y_{0}}$ & 0.48 & 0.48 & 0.39 & 0.46 & 0.44 & 0.46 \\
\hline \hline
\end{tabular}

ticity layers never extend further than two acoustic displacements away from the stack edge (indicated by a dashed line in Fig. 1). An acoustic displacement $d_{\mathrm{ac}}(x)=u_{\mathrm{ac}}(x) / \omega$ is the distance a fluid particle can travel during an acoustic half-period.

Dimensionless numbers are calculated in order to provide a better insight into the phenomenon of vortex shedding behind the stack. We define a Reynolds number Re based on viscous boundary layer thickness and velocity amplitude inside the channels of the stack, as proposed by Merkli and Thomann (1975):

$$
\operatorname{Re}=\sqrt{2} \frac{u_{\mathrm{ac}}}{\mathrm{BR}} \frac{\delta_{\nu}}{\nu}
$$

where $\nu$ is the kinematic viscosity of air. The viscous boundary layer thickness $\delta_{\nu}$ and blockage ratio BR are given in Table 1 for each configuration. Merkli and Thomann (1975) found that onset of turbulence in oscillating flows occurs when $\mathrm{Re} \geqslant 400$ approximately. The highest Reynolds number achieved during these experiments is $R e \approx 198$. As it is well below the critical Reynolds number the flow remains laminar, even though vortex shedding occurs. Reynolds numbers corresponding to the onset of vortex shedding $\mathrm{Re}_{\text {onset }}$ are presented in Table 2 . $\mathrm{Re}_{\text {onset }}$ is the Reynolds number calculated at the lowest acoustic pressure level that was tested for which vortex shedding occurs. We consider that shedding occurs when at least two consecutive pairs of counter-rotating vortices detach from the stack. According to the collected data, there is no critical Reynolds number value for the onset of oscillation.

Different Strouhal numbers are also presented in Table 2. Strouhal numbers are defined as

$$
\mathrm{St}_{D}=\frac{f_{\text {shed }} D}{u_{\text {conv }}}=\frac{D}{\Delta x}
$$

where $f_{\text {shed }}$ is the shedding frequency, $u_{\text {conv }}$ is the convection velocity of vortices, $\Delta x$ is the distance between two consecutive pairs of vortices, and $D$ is one of the characteristic lengths $\delta_{v}$, $e_{0}$ and $y_{0}$. For each configuration, Strouhal numbers are averaged over all the phase-averaged velocity fields corresponding to this configuration. We observe that the Strouhal number does not depend on the Reynolds number for a given stack configuration. It is noticeable that Strouhal numbers calculated with $D=y_{0}$ take very close values, $\mathrm{St}_{y_{0}} \approx 0.44 \pm 10 \%$, for the different configurations tested in this study.

Another approach would be to estimate the formation number $T_{\text {onset }}^{*}=2 d_{\mathrm{ac}} / y_{0}$ corresponding to the onset of vortex shedding. Indeed, in the case of vortex rings, it has been demonstrated that the detachment of the head vortex from the outflowing jet always occurs at $T^{*}$ $=4$ (Gharib et al., 1998; Zhao et al., 2000). But, from the measurements reported in Table 2, no such universal value could be found. A more extensive study covering a larger range of geometries would be required to obtain more general laws between the geometry of the stack and the flow behind it, and possibly define a criterion for the onset of shedding.

\subsection{Loss of periodicity of the flow in the gap between two facing stacks due to vortex shedding}

An analytical model (Gusev et al., 2000) and recent computational studies (Marx and BlancBenon, 2004; Besnoin and Knio, 2004) have demonstrated that a gap between the stack and 


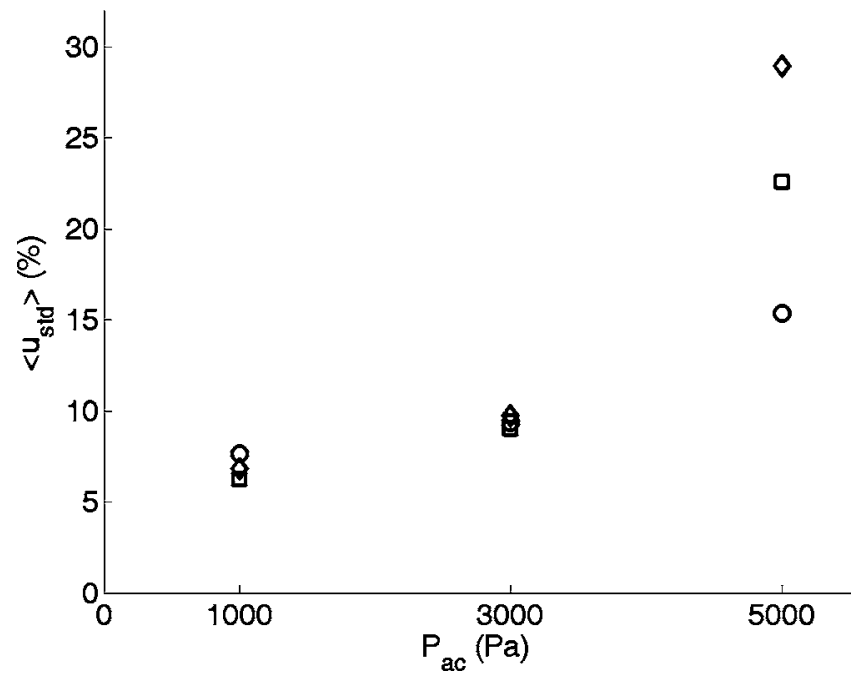

Fig. 2. Standard deviation averaged along an acoustic period and over the gap area at different acoustic pressure levels. $\bigcirc: g=0.7 \mathrm{~mm}, \square: g=1.3 \mathrm{~mm}$, and $\diamond: g=2.3 \mathrm{~mm}$.

both heat exchangers (hereafter referred to as "the gap") is favorable to the performances of the thermoacoustic system. Mm. 2 shows vorticity fields and $\Gamma_{2}=2 / \pi$ contours of the flow in the gap along an acoustic period, for four cases corresponding to $P_{\mathrm{ac}}=1000 \mathrm{~Pa}$ with gap widths $g /\left(2 d_{\mathrm{ac}}\right)=0.8$ and 2.6 , and $P_{\mathrm{ac}}=5000 \mathrm{~Pa}$ with gap widths $g /\left(2 d_{\mathrm{ac}}\right)=0.2$ and 0.5 . The hot side of the stack represented on the left in Mm. 2 is located at $x_{h}=65 \mathrm{~mm}$. Measurements are phaseaveraged over 50 images.

$\mathrm{Mm} 2$ : Vorticity fields $\Omega\left(\mathrm{s}^{-1}\right)$ and $\Gamma_{2}=2 / \pi$ contours in the gap along an acoustic period (from $\Phi$
$=0^{\circ}$ to $\left.360^{\circ}\right)$. Sampling frequency: $1 / 22.5^{\circ}$ except between $\Phi=135^{\circ}$ and $315^{\circ}$ where sampling
frequency is doubled. Top: $P_{\mathrm{ac}}=1000 \mathrm{~Pa}$, bottom: $P_{\mathrm{ac}}=5000 \mathrm{~Pa}$, left: $g=0.7 \mathrm{~mm}$, right: $g$
$=2.3 \mathrm{~mm}$. Note that the color scales are different for improved readability. The black dashed line
represents the distance $2 d_{\mathrm{ac}}$ away from the plate edge. This is a file of type "avi" (5.9 Mbytes).

At $P_{\mathrm{ac}}=1000 \mathrm{~Pa}$, vortex shedding does not occur. When $g>2 d_{\mathrm{ac}}$, a fluid particle flowing outside one stack never reaches the other stack and the flow is not disturbed by the presence of another stack. When $g<2 d_{\mathrm{ac}}$, the boundary layers flowing from one stack channel extend through the gap toward the facing channel of the other stack. The flow behaves as if there were virtual stack plates in the gap. At $P_{\mathrm{ac}}=5000 \mathrm{~Pa}$, vortex shedding occurs in the gap and the flow is similar to the flow behind a single stack described in Sec. 3.1. As Besnoin and Knio (2004) have shown, the disturbance of the flow within the gap will affect heat transfer between the stack and the heat exchangers.

We calculate the relative standard deviation of velocity measurements $u_{\text {std }}$ that we define as

$$
u_{\mathrm{std}}(x)=\frac{1}{u_{\mathrm{ac}}(x)}\left[\frac{1}{N} \sum_{n=1}^{N}\left(\bar{u}(x)-u_{n}(x)\right)^{2}\right]^{1 / 2},
$$

where $\bar{u}(x)$ is the phase-averaged velocity calculated over $N=50$ instant velocity measurements $u_{n}(x)$ performed for each phase. By definition, since measurements are phase-locked, a perfectly periodic phenomenon has zero standard deviation. Figure 2 shows velocity relative standard deviation averaged along an acoustic period and over the gap area $\left\langle u_{\text {std }}\left(x_{h}\right)\right\rangle$ for three different acoustic pressure levels and various gap widths. Relative standard deviation is approximately $6.5 \%$ and $9.5 \%$ at $P_{\mathrm{ac}}=1000 \mathrm{~Pa}$ and $P_{\mathrm{ac}}=3000 \mathrm{~Pa}$, respectively. The flow is quite repeatable from one acoustic period to another at low acoustic pressure level, regardless of the 
gap width. At high amplitudes $P_{\mathrm{ac}}=5000 \mathrm{~Pa}$, relative standard deviation increases with gap width, attaining $\left\langle u_{\text {std }}\left(x_{h}\right)\right\rangle=29 \%$ for $g / 2 d_{\mathrm{ac}}=0.5$. The flow is no longer periodic. This confirms the results obtained numerically by Besnoin (2001). As the flow within the gap does not repeat from one acoustic period to another, the average heat transfer through the gap is no longer steady, and oscillation of the cooling load will occur at high driving amplitudes.

\section{Conclusion}

This study shows the nonperiodic character of the flow within the gap between two coupled stacks as a model for a stack-heat exchanger couple in a thermoacoustic refrigerator driven at high amplitudes. PIV measurements are first conducted behind a single stack where vortex shedding is observed at high acoustic pressure level. The estimation of dimensionless numbers highlights instructive features of this phenomenon that should help in defining a criterion for the onset of vortex shedding in further studies. Standard deviation of velocity measurements shows that the flow between two coupled stacks is not repeated from one acoustic period to another at high acoustic pressure level. This loss of periodicity will lead to the oscillation of cooling load, as Besnoin (2001) has shown. Future works will include a more extensive study of the onset of oscillation of vorticity layers behind the stack and heat flux measurements on heat exchangers.

\section{Acknowledgments}

Part of this work is supported by ANR (Project MicroThermoAc NT051_42101). The authors are also grateful to N. Grosjean and J.-M. Perrin for their technical assistance and to M. Michard for his helpful advice on PIV data analysis.

\section{References and links}

Berson, A., Michard, M., and Blanc-Benon, Ph. (2007). "Measurement of acoustic velocity in the stack of a thermoacoustic refrigerator using Particle Image Velocimetry," Heat and Mass Transfer, in press.

Besnoin, E. (2001). "Numerical study of thermoacoustic heat exchangers," Ph.D. thesis, The Johns Hopkins University, Baltimore, MD.

Besnoin, E., and Knio, O. (2004). "Numerical study of thermoacoustic heat exchangers," Acta. Acust. Acust. 90, 432-444.

Blanc-Benon, Ph., Besnoin, E., and Knio, O. (2003). "Experimental and computational visualization of the flow field in a thermoacoustic stack," C. R. Mec. 331, 17-24.

Duffourd, S. (2001). "Réfrigérateur thermoacoustique: Études analytiques et expérimentales en vue d'une miniaturisation," Ph.D. thesis. 2001-06, Ecole Centrale de Lyon (in French), Ecully, France. ("Thermoacoustic refrigerator: Analytical and experimental studies with the prospect of miniature systems".)

Gharib, M., Rambod, E., and Shariff, K. (1998). “A universal time scale for vortex ring formation,” J. Fluid

Mech. 360, 121-140.

Graftieaux, L., Michard, M., and Grosjean, N. (2001). "Combining PIV, POD and vortex identification

algorithms for the study of unsteady turbulent swirling flows," Meas. Sci. Technol. 12, 1422-1429.

Gusev, V., Lotton, P., Baillet, H., Job, S., and Bruneau, M. (2000). "Relaxation-time approximation for analytical evaluation of temperature field in a thermoacoustic stack," J. Sound Vib. 235, 711-726.

Marx, D. (2003). "Simulation numérique d'un réfrigérateur thermoacoustique," Ph.D. thesis. 2003-34, Ecole Centrale de Lyon (in French), Ecully, France. ("Numerical simulation of a thermoacoustic refrigerator".) Marx, D., and Blanc-Benon, Ph. (2004). "Numerical simulation of the stack-heat exchangers coupling in a thermoacoustic refrigerator," AIAA J. 42, 1338-1347.

Merkli, P., and Thomann, H. (1975). "Transition to turbulence in oscillating pipe flow," J. Fluid Mech. 68, 567-575.

Poese, M., and Garrett, S. (2000). "Performance measurements on a thermoacoustic refrigerator driven at high amplitudes," J. Acoust. Soc. Am. 107, 2480-2486.

Smith, B., and Swift, G. (2003). "Power dissipation and time-averaged pressure in oscillating flow through a sudden area change," J. Acoust. Soc. Am. 113, 2455-2463.

Wakeland, R., and Keolian, R. (2002). "Influence of velocity profile nonuniformity on minor losses for flow exiting thermoacoustic heat exchangers," J. Acoust. Soc. Am. 112, 1249-1252.

Waxler, R. (2001). "Stationary velocity and pressure gradients in a thermoacoustic stack," J. Acoust. Soc. Am. 109, 2739-2750.

Wetzel, M., and Herman, C. (2000). "Experimental study of thermoacoustic effect on a single plate. I.

Temperature fields," Heat Mass Transfer 36, 7-20.

Zhao, W., Frankel, S., and Mongeau, L. (2000). "Effects of trailing edge instability on vortex ring formation,"

Phys. Fluids 12, 589-596. 